\title{
A Geometrical origin of the Pulsar Core and Conal Emissions
}

\section{R.C.Kapoor}

Indian Institute of Astrophysics, Koramangala, Bangalore 560034, India

\section{C.S.Shukre}

Raman Research Institute, Sadashivanagar, Bangalore 560080, India

We have analysed the dipole magnetic field geometry for the general case of an oblique rotator and have found that open field lines which define the polar cap divide into two branches (Kapoor and Shukre 1996) which appear naturally relevant for distinguishing the core and conal emissions. The polar cap shape is actually determined by a quadratic equation having two roots leading to two values of the polar angle, $\theta^{+}$and $\theta^{-}$with respect to the magnetic axis for a given azimuth $\varphi$. For the north pole both $\theta^{+}$and $\theta^{-}$branches are shown as polar plots in Fig. 1 for various inclination angles $\alpha$ and a typical pulsar period $P=1 / \sqrt{2} s$. The discussion of pulsar polar caps hitherto (e.g. Biggs 1990) had not distinguished between the $\theta^{+}$and the $\theta^{-}$solutions. The region defined by the $\theta^{+}$solution is completely contained inside the polar cap. It has a peculiar triangular shape whose lowest vertex is always on the magnetic axis. This naturally suggests an identification of the $\theta^{+}$and the $\theta^{-}$regions with the core and conal emission zones. This idetification leads to following consequences:
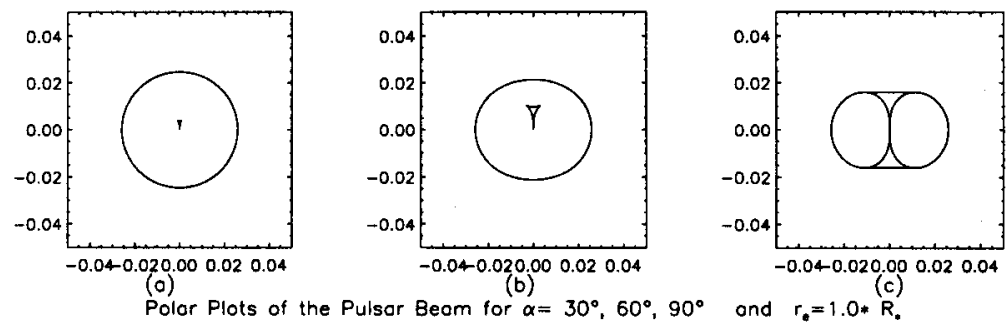

Figure 1.

As is seen from Fig. 1, for the core component to have a width comparable to that of the conal component as is observed, its emission height, $r_{\text {core }}$, must be much greater than that of the conal emission, $r_{\text {cone }}$. A previous suggestion (Rankin 1990) that core emission originates on the stellar surface runs into difficulty if light bending effects are included. Our proposal circumvents this difficulty. With increasing inclination angle $\alpha$, the conal emission zone displays a latitudinal compression (as noted by others earlier, e.g.', Biggs 1990) whereas the core emission zone displays a latitudinal elongation. This elongation may be the one discussed earlier (Narayan and Vivekanand 1983). Higher altitudes for the emission of core components imply a lead with respect to the conal components due to aberration of light. This permits determination of $\alpha$ using 
the observed separation between core and conal centres, e.g., for PSR $1917+00$ we predict $\alpha \simeq 75^{\circ}$, in agreement with Lyne and Manchester (1988) and Rankin (1993). Various testable predictions can be made about interpulses.

Our picture of pulsar core and conal emissions is not only consistent with observations but also removes some disagreements between previous analyses. A reanalysis of observations in the light of our proposal is required to test it further and if possible to derive constraints on possible dynamics of emission.

\section{References}

Biggs J.D., 1990, M N R A S, 245, 514

Kapoor R.C. \& Shukre C.S., 1996, (to be published)

Lyne A.G. \& Manchester R.N., 1988, M N R A S, 234,477

Narayan R. \& Vivekanand M.,1983, Astr. \& Ap. 122, 45

Rankin J., 1990, Ap.J., 352, 247.

Rankin J., 1993, Ap.J., 405, 285. 